\title{
Reliability of specific on-ice repeated-sprint ability test for ice-hockey players
}

\author{
Karel Hůlka*, Jan Bělka, Roman Cuberek, and Ondřej Schneider \\ Faculty of Physical Culture, Palacký University, Olomouc, Czech Republic
}

Copyright: (C) 2014 K. Hůlka et al. This is an open access article licensed under the Creative Commons Attribution License (http:// creativecommons.org/licenses/by/4.0/).

\begin{abstract}
Background: Repeated sprint ability tests are today widely used to evaluate the performance capability in team sports. Objective: The aim of this study was to determine the reliability of a specific ice hockey test, which indicates the agility and repeated-sprint ability of the players. Methods: Twenty four highly trained junior ice hockey players (age $=17.68 \pm 1.52$ years; $\left.B M I=23.8 \pm 1.92 \mathrm{~kg} \cdot \mathrm{m}^{-2}\right)$ participated in the study. Each participant was assessed for specific on-ice repeated-sprint ability test $12 \times 54 \mathrm{~m}$ with $30 \mathrm{~s}$ rest. Intraclass correlation coefficient (association between two repeated measurements) and coefficient of variation were calculated to assess the reliability of the test. Results: All intraclass correlation coefficients were .78 for sprint decrement and .98 for total time and the best time, the coefficient of variation was $1.52 \%$ for best sprint time, $1.31 \%$ for total time and $19.3 \%$ for sprint decrement variable. Conclusions: The results suggest the high reliability of the ice hockey agility test expressed by the best sprint time and repeated-sprint ability by the total time and less reliability of sprint decrement.
\end{abstract}

Keywords: intermittent exercise, sprint decrement, specificity, recovery

\section{Introduction}

Contemporary training process has witnessed a great deal of changes due to increasing scientific and expert knowledge about the nature of conditioning in team sports. The specificity of the physical training becomes a key factor in planning the training process, because of need to attain specific physiological adaptations as much as possible. That is why we should analyse the demands of the game by time motion analysis (Reilly, 2005) and adequately operate with a player's load intensity and activities during the training process.

Time motion analysis research has pointed out ice hockey as a high speed intermittent invasion game similar to the other games (Buchheit, Lefebvre, Laursen, \& Ahmaidi, 2011; Montgomery, 1988). The game is characterised by irregular changes between high and low intensity activities over very short periods of time (up to $10 \mathrm{~s}$ ) with high-impact body contact. Low intensity activities are related to the recovery processes (Balsom, Seger, Sjodin, \& Ekblom, 1992; Quinney et al., 2008; Vescovi, Murray, \& VanHeest, 2006). Each on-ice shift last between 30 and $80 \mathrm{~s}$ interspersed with 2 to $5 \mathrm{~min}$ of recovery between shifts (Arnett, 1996; Quinney et al.,

*Address for correspondence: Karel Hůlka, Department of Sports, Faculty of Physical Culture, Palacký University, tř. Míru 115, 77111 Olomouc, Czech Republic. E-mail: karel.hulka@upol.cz
2008). Noonan (2010) determined the work rest ratio to 0.43 . The physiological profile of elite ice hockey players require both aerobic and anaerobic metabolism for example Twist and Rhodes (1993) stated $60-70 \%$ of used energy form aerobic metabolism. Noonan (2010) found out that the blood lactate was ranged from 4.4 to $13.7 \mathrm{mmol} \cdot \mathrm{l}^{-1}$ during ice hockey match. Montgomery (1988) and Cox, Miles, Verde, and Rhodes (1995) found the heart rates during a shift on the ice exceed $90 \%$ of maximal heart rate $\left(\mathrm{HR}_{\max }\right)$ with average on-ice values of about $85 \%$ of $\mathrm{HR}_{\max }$.

The ability to quickly replenish energy stores during short and active rest in the working muscles is critical for ice hockey performance (Arnett, 1996; Montgomery, 1988) this is called the repeated-sprint ability - RSA (Girard, Mendez-Villanueva, \& Bishop, 2011). RSA-based exercises are characterized by repeated sprints interspersed with brief recovery periods. Such exercise results similar metabolic responses as those which occur during actual matches, such as decrease in a muscle $\mathrm{pH}$, phosphocreatine and ATP, activation of anaerobic glycolysis and significant involvement of the aerobic metabolism (Rampinini et al., 2007; Wragg, Maxwell, \& Doust, 2000). For this reason, the use of RSA-based exercises during the training and for testing of team sports athletes is increasing (Spencer, Bishop, Dawson, \& Goodman, 2005).

To respect a new specific training demands, which are based on the game needs, it is important to develop 
a new performance test tools. Therefore tests of multiple sprints performance or repeated-sprint ability are becoming increasingly popular as a way of evaluating the performance capabilities of athletes involved in field and court sports (Glaister et al., 2009).

Some authors who work with RSA tests use work/rest ratio, sprint number and duration as a background for creating RSA tests (Alizadeh, Hovanloo, \& Safania, 2010; Meckel, Gottlieb, \& Eliakim, 2009; Psotta, Blahus, Cochrane, \& Martin, 2005). Another authors add agility parameters (as changes of direction and locomotion) to sprint activity (Aguiar et al., 2008; da Silva, Guglielmo, \& Bishop, 2010; Wragg, Maxwell, $\&$ Doust, 2000) for better simulation of game demands.

For ice hockey use (Leone, Leger, Lariviere, \& Comtois, 2007) a multistage $45 \mathrm{~m}$ aerobic test with stop and go called SMART (Skating Maximal Aerobic Test) was made. Petrella, Montelpare, Nystrom, Plyley, and Faught (2007) created another multistage test called FAST (48.8 meters), but without full ice hockey equipment. Buchheit, Lefebvre, Laursen, and Ahmaidi (2011) created on-ice 30-15 intermittent test similar to original 30-15 intermittent running test. All these tests contain only forward skating with stops.

Power, Faught, Przysucha, McPherson, and Montelpare (2012) designed and validated the high intensity anaerobic intermittent tests with very short work and rest intervals for ice hockey players called Repeat Ice Skating Test (RIST), where the participants completed $6 \times 49$ m circles with 10s recovery between trials. As a first Reed et al. (1979) published this type of test called Reed Repeat Sprint Test (RRS) consists of $6 \times 91 \mathrm{~m}$ sprints with $30 \mathrm{~s}$ recovery. This test protocol was criticized for being too exhausting (Nightingale, Miller, \& Turner, 2012). Carey, Drake, Pliego, and Raymond (2007) used the test protocol with $5 \times 1$ lap sprints with a $30 \mathrm{~s}$ recovery period between laps.

We have constructed the specific on-ice repeated ability test (RAT) for ice hockey players. In this RAT we have tried to simulate the game demands on ice hockey players. Firstly, according to Léger, Seliger, and Bassard (1979) who showed lower mechanical efficiency $(7.9 \%)$ of runners compare to skaters during the test is important to get valid data about repeatedsprint ability test for skaters. Secondly there is no evidence about performance test which is constructed to indicate repeated-sprint ability as well as use of the agility patterns (starts, repeated sprints with change of direction while sprinting, stops, and different types of skating) during the same sprint activity as during the ice hockey game. The main difference among our constructed protocol and the others test protocols (Carey, Drake, Pliego, \& Raymond; Power et al., 2012; Reed et al., 1979) we see in higher specificity of the load thanks to recovery time, shorter sprints, more changes of direction, and different types of skating during each trial. In addition, before using the new performance test effectively, the researchers should determine reliability of the measurement Spencer, Fitzsimons, Dawson, Bishop, and Goodman (2006).

Many authors (Oliver, Williams, \& Armstrong, 2006; Psotta, Bunc, Hendl, Tenney, \& Heller, 2011) found the reliability of fatigue index as moderate. The reason can be pacing effect (Oliver, 2009) or small familiarisation (Oliver, 2007). According to Oliver (2007) we chose the sprint decrement as an indicator of fatigue. For reliability measurement design we followed by recommendations of Atkinson and Nevill (1998) and Hopkins, Schabort, and Hawley (2001), and used indicators of relative (intraclass correlation coefficient) and absolute reliability (coefficient of variation and Bland and Altman's 95\% limits of agreement). The aim of this study was to construct and determine the reliability of the specific ice hockey test indicating repeated-sprint ability of the players.

\section{Methods}

\section{Participants}

Twenty four highly trained junior ice hockey players from one team of the highest junior division in the Czech Republic (age $=17.7 \pm 1.5$ years; height $=174.5 \pm 6.1 \mathrm{~cm}$; weight $=72.5 \pm 6.8 \mathrm{~kg}$; $\mathrm{BMI}=$ $=23.8 \pm 1.9 \mathrm{~kg} \cdot \mathrm{m}^{-2}$ ) volunteered to participate in the present study. The players trained on average five times a week plus matches. The aims and objectives of the present study have been clarified to all participants and all of them gave a voluntary written informed consent. Participation was voluntary and players could withdraw from the study at any time. Goalkeepers were excluded from the reliability analysis because of their entirely different type of performance during the ice hockey match.

\section{Procedures}

All participants completed two repetitive measurements of the specific repeated-sprint ability test for ice hockey with the full ice hockey equipment. Two measurements were made one week apart always after day off. The research was held after the regular competition season. All trials were performed at the same day-time to minimise the effect of diurnal variability. The subjects were instructed not to take any food two hours before testing. All measurements were held at an ice hockey arena with standardized size of the ice hockey rink $(60 \times 28 \mathrm{~m})$. 


\section{Specific repeated-sprint ability test protocol}

A week before the testing all participants were required to perform trial sprints to become familiar with the track and two sprints to determine the length of the recovery period between the sprints. To develop good specific RSA test, it is necessary to know the work rate profiles of players during the match (Psotta, Blahus, Cochrane, \& Martin, 2005). The work rest ratio was stated from the results of time motion analysis (Noonan, 2010) to work/rest ratio $=0.43$. The average sprint time of the trials was $12.74 \pm 0.78 \mathrm{~s}$ and recovery period was determined by $30 \mathrm{~s}$.

The average duration of one short sprint during the match was from 2.0 to $3.5 \mathrm{~s}$ (Green et al., 1976). That is why three changes of direction were set. Standard warm up procedures on both test days consisted of 15 minutes active warm up and thereafter five short sprints (length of the court) followed by five minute recovery time during which was done the test protocol description.

The participants were required to perform maximal $12 \times 80 \mathrm{~m}$ sprint trials according to Figure 1 . One trial consisted of $18 \mathrm{~m}$ forward skating finished by stopping on the blue lane ( $2 \mathrm{~m}$ from barrier), followed by $22 \mathrm{~m}$ backward skating by stopping on the goal lane, then
$22 \mathrm{~m}$ forward skating finished by sharp turn around the cone on the blue lane ( $2 \mathrm{~m}$ from barrier) and the last $18 \mathrm{~m}$ forward skating to the finished line. All trials were recorded using electronic timing gates and the recovery time using hand stop watches.

Three variables from the raw data were calculated: the best sprint time (s), sprint decrement (\%) as an indicator of repeated-sprint ability and total time (s). Sprint decrement $\left(\mathrm{S}_{\mathrm{dec}}\right)$ was calculated according to Girard, Mendez-Villanueva, and Bishop (2011):

$$
\mathrm{S}_{\mathrm{dec}}(\%)=\left(\frac{\mathrm{S}_{1}+\mathrm{S}_{2}+\ldots+\mathrm{S}_{12}}{10 \cdot \mathrm{S}_{\text {best }}}-1\right) \cdot 100,
$$

where $S_{1-12}$ are times of ten single sprints, $S_{\text {best }}$ is the best time from the single sprints. Total time was calculated as a sum of twelve single sprint times.

\section{Statistical analyses}

SPSS software (Version 17; SPSS Inc., Chicago, IL, USA) was used to process the data. Descriptive statistic mean and standard deviation was used to describe participant's performance during the test. The homoscedasticity was assessed by using the Pearson product moment correlation coefficient (the absolute difference

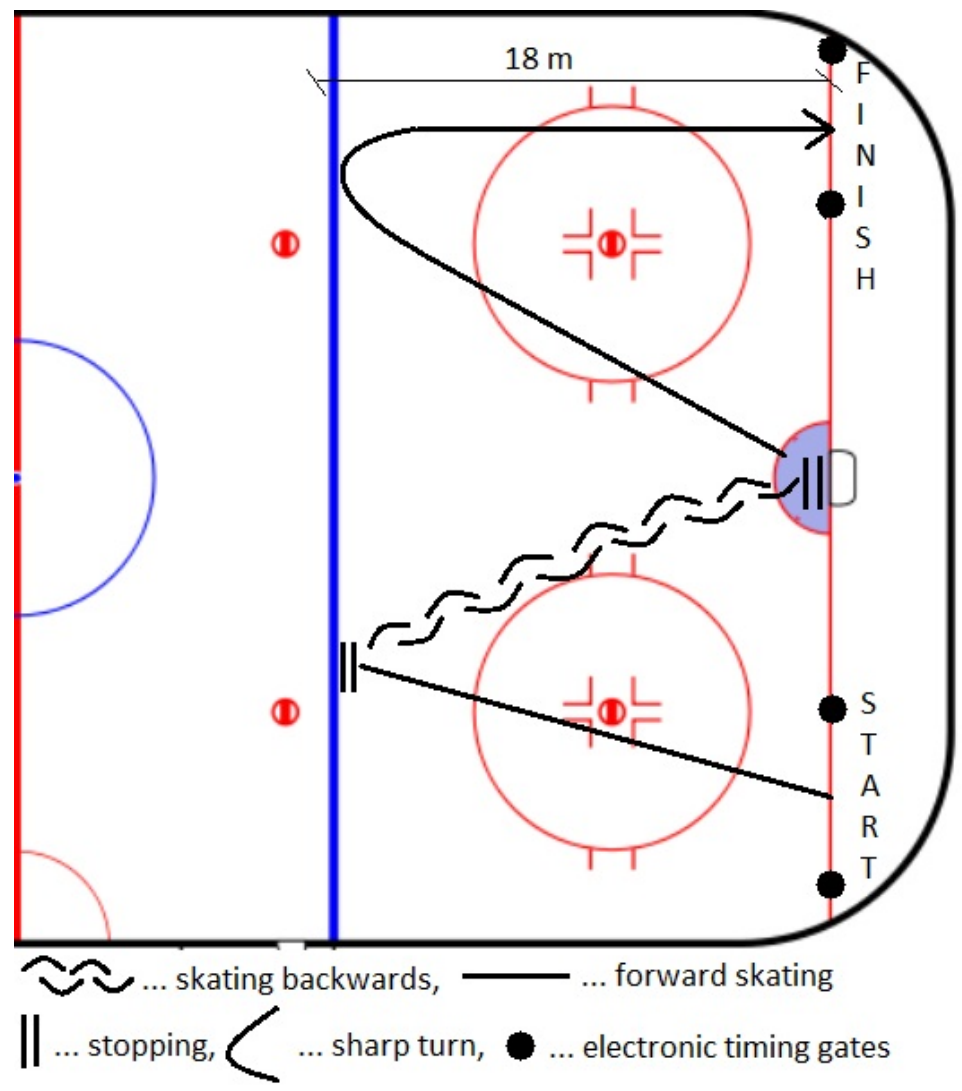

Figure 1. Graphic illustration of the specific on-ice repeatedsprint ability test 
between two repeated assessments vs. the mean of the two repeated assessments). The intraclass correlation coefficient $I C C=(M S S-M S E) \cdot M S^{-1}$, where $M S S$ is the mean square of the subjects, MSE is the mean square of the error, and $M S$ is the mean square, was calculated as an indicator of intra-subject reliability. The coefficient of variation and Bland and Altman's 95\% limits of agreement for two repeated measurements were used to express and assess the reliability (absolute reliability) in the original measurement unit (Atkinson \& Nevill, 1998). The coefficient of variation $(\mathrm{CV})$ was calculated as a percentage expression of typical error of measurement (TEM) according to Hopkins, Schabort, and Hawley (2001). To calculate TEM, the following formula was used (Hopkins et al., 2001): $T E M=S_{\text {diff }} / \sqrt{2}$, where $S_{\text {diff }}$ is the standard deviation of difference score between two measurements.

To determine the reliability of changes of times during the all tests the standard error of measurement (SEM) was used. To calculate the SEM, the following formula was used (Thomas, Nelson, \& Silverman, 2005): $S E M=S D \cdot \sqrt{ }(1-I C C)$ where $S D$ is the standard deviation of the sample and ICC is the calculated intraclass correlation coefficient (the unbiased error). The ICC was calculated from the differences $\left(D_{\mathrm{i}}\right)$ between average times of each trial $\left(X_{\mathrm{i}}\right)$ in first and second measurement by $D_{i}=X_{i+1}-X_{i}$, where $i=1,2, \ldots, 10$. The statistical significances of all parts of the analysis were determined at an alpha level of $p<.05$.

\section{Results}

In the first measurement the mean of the total time from ten sprints (TT) was $129.8 \pm 9.9 \mathrm{~s}$, the mean of the best sprint time (BT) was $12.45 \pm 0.9 \mathrm{~s}$ and the mean of the sprint decrement $5.4 \pm 1.7 \%$ (opposite) compare to $\mathrm{TT}=131.5 \pm 11.2 \mathrm{~s}, \quad \mathrm{BT}=12.43 \pm 0.9 \mathrm{~s}$ and $\mathrm{S}_{\mathrm{dec}}=4.7 \pm 1.3 \%$ from the second measurement. The individual times are presented in Table 1.

Table 2 presents the results of the reliability analysis. The results of correlation analysis show high relative reliability of the repeated measurements. Especially the high value of the ICC indicates very high level of intrasubject reliability in all monitored variables. Similarly Pearson's correlation coefficient is high in all variables. It indicates a high association between the results of both repeated measurements.

Similar tendency show the indicators of absolute reliability. The change in the mean was minimal in all variables. The participants achieved better values in BT and $\mathrm{S}_{\mathrm{dec}}$ in the second measurement, and TT had negative tendency. Coefficient of variation was 1.52\% for total sprint time, $0.19 \%$ for best time and $0.69 \%$ for
Table 1

Individual times of participants during all measurements

\begin{tabular}{|c|c|c|c|c|c|c|}
\hline Participant & BEST1 & BEST2 & TT1 & TT2 & $\mathrm{S}_{\mathrm{dec} 1}$ & $\mathrm{~S}_{\mathrm{dec} 2}$ \\
\hline 1 & 12.43 & 12.51 & 129.52 & 130.74 & 5.18 & 3.53 \\
\hline 2 & 12.77 & 12.83 & 131.43 & 135.03 & 5.74 & 3.44 \\
\hline 3 & 12.78 & 12.89 & 131.64 & 133.25 & 4.26 & 3.13 \\
\hline 4 & 13.00 & 12.81 & 132.95 & 134.02 & 3.09 & 3.79 \\
\hline 5 & 13.42 & 13.21 & 138.42 & 138.81 & 3.44 & 4.78 \\
\hline 6 & 12.71 & 12.87 & 132.95 & 134.11 & 5.52 & 3.30 \\
\hline 7 & 13.67 & 13.66 & 145.31 & 149.65 & 9.47 & 7.88 \\
\hline 8 & 13.77 & 13.51 & 146.20 & 150.30 & 9.15 & 8.22 \\
\hline 9 & 12.57 & 13.12 & 134.62 & 133.48 & 6.19 & 4.61 \\
\hline 10 & 13.54 & 13.54 & 142.37 & 141.23 & 4.31 & 5.15 \\
\hline 11 & 13.80 & 13.93 & 146.07 & 154.03 & 6.62 & 4.86 \\
\hline 12 & 13.80 & 13.90 & 145.79 & 150.77 & 6.25 & 4.88 \\
\hline 13 & 12.29 & 11.87 & 124.34 & 126.09 & 3.00 & 4.05 \\
\hline 14 & 11.90 & 11.85 & 123.24 & 126.27 & 5.61 & 4.00 \\
\hline 15 & 11.34 & 11.54 & 119.19 & 118.57 & 4.56 & 3.28 \\
\hline 16 & 11.12 & 11.49 & 118.92 & 118.03 & 6.14 & 5.00 \\
\hline 17 & 11.39 & 11.29 & 119.31 & 120.99 & 6.22 & 5.68 \\
\hline 18 & 11.63 & 11.14 & 117.18 & 118.78 & 4.13 & 5.19 \\
\hline 19 & 11.11 & 11.08 & 116.01 & 118.01 & 6.22 & 4.70 \\
\hline 20 & 11.21 & 11.57 & 122.04 & 119.74 & 6.82 & 5.48 \\
\hline 21 & 11.99 & 11.88 & 124.80 & 127.18 & 6.07 & 5.05 \\
\hline 22 & 11.99 & 11.87 & 125.54 & 126.90 & 5.84 & 5.76 \\
\hline 23 & 12.13 & 11.94 & 123.63 & 124.97 & 3.03 & 3.54 \\
\hline 24 & 12.38 & 11.99 & 124.51 & 125.24 & 3.16 & 3.84 \\
\hline Mean & 12.45 & 12.43 & 131.51 & 129.83 & 5.42 & 4.71 \\
\hline$S D$ & 0.88 & 0.89 & 10.92 & 9.67 & 1.69 & 1.28 \\
\hline
\end{tabular}

Note. BEST1 $=$ best time in the first measurement, BEST2 $=$ best time in the second measurement, TT1 = total time in the first measurement, TT2 = total time in the second measurement, $S_{\text {dec } 1}=$ sprint decrement in the first measurement, $\mathrm{S}_{\mathrm{dec} 2}=$ sprint decrement in the second measurement.

sprint decrement. Similarly low differences were found between results expressed by Bland and Altman's 95\% limits of agreement which refer to $\pm 4.77 \mathrm{~s}, \pm 0.53 \mathrm{~s}$ and $\pm 1.91 \%$ respectively.

The results of standard error of measurement SEM as an indicator of the reliability of changes of times during the all test was $0.11 \mathrm{~s}$. It represented only $0.88 \%$ of the average measured time (13.07 s).

\section{Disscussion}

Reliability is a theoretical concept that is used to describe the quality of a measurement instrument. The aim of this study was to assess the reliability of 
Table 2

Overview of reliability analysis indicators

\begin{tabular}{|c|c|c|c|}
\hline Reliability procedures/level of an indicator & TT $(\mathrm{s})$ & BT (s) & $\mathrm{S}_{\mathrm{dec}}(\%)$ \\
\hline \multicolumn{4}{|l|}{ Change of the mean of two trials } \\
\hline Mean $_{\text {diff }}+s_{\text {diff }}$ & $1.68 \pm 2.25$ & $-0.02 \pm 0.25$ & $-0.70 \pm 1.15$ \\
\hline \multicolumn{4}{|l|}{ Retest correlation } \\
\hline ICC & .97 & .98 & .78 \\
\hline $95 \% \mathrm{CI}$ & $(.96, .99)$ & $(96, .99)$ & $(.50, .91)$ \\
\hline$R$ & .98 & .96 & .74 \\
\hline \multicolumn{4}{|l|}{ Within-subject variation } \\
\hline TEM & 1.72 & 0.19 & 9.69 \\
\hline CV & $1.31 \%$ & $1.52 \%$ & $19.3 \%$ \\
\hline $95 \%$ limits of agreement & $\pm 4.77 \mathrm{~s}$ & $\pm 0.53 \mathrm{~s}$ & $\pm 1.91 \%$ \\
\hline
\end{tabular}

Note. $\quad \mathrm{TT}=$ total time, $\mathrm{BT}=$ best time, $\mathrm{S}_{\mathrm{dec}}=$ sprint decrement. Mean ${ }_{\text {diff }}=$ mean of difference between test-retest measurements, $\mathrm{s}_{\text {diff }}=$ standard deviation of difference between test-retest measurements, $I C C=$ intraclass correlation coefficient, $95 \% \mathrm{CI}=95 \%$ confidence interval, $R=$ Pearson product moment correlation coefficient, $T E M=$ typical error of measurement, $C V=$ coefficient of variation.

the specific repeated-sprint ability test protocol for icehockey players.

The small differences were found between the means of two measurements. The players achieved worse result during the second measurement in TT, but better results in $\mathrm{BT}$ and $\mathrm{S}_{\mathrm{dec}}$. These changes can be explained due to the previous experience with the test (known as the learning effect). Nevertheless we consider the changes in the means of two measurements as very small and acceptable for all indicators.

To rate $\mathrm{BT}$, the relative reliability (expressed by ICC) shows very close relation between two measurements $(r=.98)$. Results are widely comparable with other repeated-sprint ability studies. Oliver (2009) presented $C V$ ranging 0.7 to $2.7 \%$ for BT. Glaister et al. (2009) presented very good reliability $r=.91$ for $40 \mathrm{~m}$ Shuttle run test and $r=.94$ for RMAT by Haj-Sassi et al. (2011). The within subject variation expressed by coefficient of variation show small differences between two measurements too. The $C V=1.52 \%$ shows very small error of measurement similarly as $C V=0.82 \%$ of Wragg et al. (2000) for The Maximal Anaerobic Running Test (MART). Oliver, Armstrong, and Williams (2009) recommended $C V$ for BT lower than $3 \%$. The researchers and practitioners should utilize results of Bland and Altman's 95\% limits of agreement during the interpretation of the results. Therefore it is necessary to take into account determined value of $\pm 0.53 \mathrm{~s}$. Its magnitude should be considered as negligible difference between two measurements.

The sprint trial was made as an agility pattern of ice hockey performance. We think that BT could represent an indicator of ice skating (locomotion) skills of the players. To use this, verification is needed. Our test protocol, where we put together the measurement of ice skating (locomotion) skills and repeated-sprint ability, could save the time during practice.

Assessment of fatigue was expressed by $\mathrm{S}_{\mathrm{dec}}$ as a fatigue index and TT as a global RSA performance - ability of the player to sustain his power output at high-intensity, there was a very close relation of the ICC between two measurements too. The absolute reliability values of TT seems to be high and satisfactory as the results of Haj-Sassi et al. (2011), who had gained $0.93 \%$ for RMAT test and Spencer et al. (2006) 0.70\% for field hockey test respectively. Moreover the value of $C V$ equal to $5 \%$ is referred (Buchheit et al., 2011) as a criterion of good reliability and sensitivity, Hopkins, Hawley, and Burke (1999) refer 3\%. Total time variable of our study accomplished this criterion like above mentioned studies. The ICC shows similar reliability as Boddington, Lambert, Gibson, and Noakes (2001) .98 for $5 \mathrm{~m}$ shuttle run test respectively. Similarly the results of relative reliability indicate high relationship between both measurements. We can use total time of our test protocol for repeated-sprint ability measurement.

The value of $C V$ for $\mathrm{S}_{\mathrm{dec}}$ is quite higher (19.3\%) than other variables because of small percentage of sprint decrement, but similar to Gabbett (2010) and Spencer et al. (2006) and lower than $38.7 \%$ of Glaister et al. (2009).

To reach relevant and credible results, the maximal effort of players during the measurements is needed. For the control of the effort the maximum of difference among every sprint during each measurement and the 
mean of two trial sprints a week before first measurement had to be less than $10 \%$.

The observed value of the standard error of measurement of $0.11 \mathrm{~s}$ indicates the satisfactory quality of this instrument. This value appears to be insignificant in view of the purpose of the test protocol. The SEM shows relative stability of fatigue during skating and tendency to decrease the effort of participants during the test.

As a limitation of this study we considered only two performed repeated measures and the possible changeability of the distance covered due to skating backwards.

\section{Conclusions}

In this study we proved the high reliability of our test for ice hockey players expressed by the best sprint time variable. When analysing test as specific repeatedsprint ability indicator, we found out that the total time variable was highly reliable. On the other hand, the sprint decrement variable is a less reliable indicator for repeated-sprint ability similar to other RSA test studies. Our RSA test can be used as a specific ice hockey repeated-sprint ability test on the ice.

\section{References}

Aguiar, M., Abrantes, C., Macas, V., Leite, N., Sampaio, J., \& Ibanez, J. (2008). Effects of intermittent or continuous training on speed, jump and repeated-sprint ability in semi-professional soccer players. The Open Sports Sciences Journal, 1, 15-19.

Alizadeh, R., Hovanloo, F., \& Safania, A. M. (2010). The relationship between aerobic power and repeated sprint ability in young soccer players with different levels of $\mathrm{VO}_{2}$ max. Journal of Physical Education and Sport, 27, 86-92.

Arnett, M. G. (1996). Effects of specificity training on the recovery process during intermittent activity in ice hockey. Journal of Strength and Conditioninig Research, 10, 124-126.

Atkinson, G., \& Nevill, A. M. (1998). Statistical methods for assessing measurement error (reliability) in variables relevant to sports medicine. Sports Medicine, 26, 217-238.

Balsom, P. D., Seger, J. Y., Sjodin, B., \& Ekblom, B. (1992). Maximal-intensity intermittent exercise - effect of recovery duration. International Journal of Sports Medicine, 13, 528-533.

Boddington, M. K., Lambert, M. I., Gibson, A. S. C., \& Noakes, T. D. (2001). Reliability of a 5 m multiple shuttle test. Journal of Sports Sciences, 19, 223-228.

Buchheit, M., Lefebvre, B., Laursen, P. B., \& Ahmaidi, S. (2011). Reliability, usefulness, and validity of the 30-15 intermittent ice test in young elite ice-hockey players. Journal of Strength and Conditioning Research, 25, 1457-1464.
Carey, D. G., Drake, M. M., Pliego, G. J., \& Raymond, R. L. (2007). Do hockey players need aerobic fitness? Relationship between $\mathrm{VO}_{2}$ max and fatigue during high-intensity intermittent ice skating. Journal of Strength and Conditioning Research, 21, 963-966.

Cox, M. H., Miles, D. S., Verde, T. J., \& Rhodes, E. C. (1995). Applied physiology of ice hockey. Sports Medicine, 19, 184-201.

da Silva, J. F., Guglielmo, L. G. A., \& Bishop, D. (2010). Relationship between different measures of aerobic fitness and repeated-sprint ability in elite soccer players. Journal of Strength and Conditioning Research, 24, 2115-2121.

Gabbett, T. J. (2010). The development of a test of repeatedsprint ability for elite women's soccer players. Journal of Strength and Conditioning Research, 24, 1191-1194.

Girard, O., Mendez-Villanueva, A., \& Bishop, D. (2011). Repeated-sprint ability - part I: Factors contributing to fatigue. Sports Medicine, 41, 673-694.

Glaister, M., Hauck, H., Abraham, C. S., Merry, K. L., Beaver, D., Woods, B., \& McInnes, G. (2009). Familiarization, reliability, and comparability of a $40 \mathrm{~m}$ maximal shuttle run test. Journal of Sports Science and Medicine, 8, 77-82.

Green, H., Bishop, P., Houston, M., Mckillop, R., Norman, R., \& Stothart, P. (1976). Time, motion and physiological assessments of ice-hockey performance. Journal of Applied Physiology, 40, 159-163.

Haj-Sassi, R., Dardouri, W., Gharbi, Z., Chaouachi, A., Mansour, H., Rabhi, A., \& Mahfoudhi, M. E. (2011). Reliability and validity of a new repeated agility test as a measure of anaerobic and explosive power. Journal of Strength and Conditioning Research, 25, 472-480.

Hopkins, W. G., Hawley, J. A., \& Burke, L. M. (1999). Design and analysis of research on sport performance enhancement. Medicine \& Science in Sports \& Exercice, 31, 472-485.

Hopkins, W. G., Schabort, E. J., \& Hawley, J. A. (2001). Reliability of power in physical performance tests. Sports Medicine, 31, 211-234.

Léger, L., Seliger, V., \& Brassard, L. (1979). Comparsions among $\mathrm{VO}_{2} \max$ values for hockey players and runners. Canadian Journal of Applied Sport Sciences, 4, 18-21.

Leone, M., Leger, L. A., Lariviere, G., \& Comtois, A. S. (2007). An on-ice aerobic maximal multistage shuttle skate test for elite adolescent hockey players. International Journal of Sports Medicine, 28, 823-828.

Meckel, Y., Gottlieb, R., \& Eliakim, A. (2009). Repeated sprint tests in young basketball players at different game stages. European Journal of Applied Physiology, 107, 273-279.

Montgomery, D. L. (1988). Physiology of ice-hockey. Sports Medicine, 5, 99-126.

Nightingale, S. C., Miller, S., \& Turner, A. (2013). The usefulness and reliability of fitness testing protocols for ice hockey players: A literature review. Journal of Strength and Conditioning Research, 27, 1742-1750.

Noonan, B. C. (2010). Intragame blood-lactate values during ice-hockey and their relationships to Commonly used hockey testing protocols. Journal of Strength and Conditioning Research, 24, 2290-2295. 
Oliver, J. L. (2009). Is a fatigue index a worthwhile measure of repeated sprint ability? Journal of Science and Medicine in Sport, 12, 20-23.

Oliver, J. L., Armstrong, N., \& Williams, C. A. (2009). Relationship between brief and prolonged repeated sprint ability. Journal of Science and Medicine in Sport, 12, 238-243.

Oliver, J. L., Williams, C. A., \& Armstrong, N. (2006). Reliability of a field and laboratory test of repeated sprint ability. Pediatric Exercise Science, 18, 339-350.

Petrella, N. J., Montelpare, W. J., Nystrom, M., Plyley, M., \& Faught, B. E. (2007). Validation of the FAST skating protocol to predict aerobic power in ice hockey players. Applied Physiology, Nutrition, and Metabolism, 32, 693-700.

Power, A., Faught, B. E., Przysucha, E., McPherson, M., \& Montelpare, W. (2012). Establishing the test-retest reliability and concurrent validity for the repeat ice skating test (RIST) in adolescent male ice hockey players. Measurement in Physical Education and Exercise Science, 16, 69-80.

Psotta, R., Blahus, P., Cochrane, D. J., \& Martin, A. J. (2005). The assessment of an intermittent high intensity running test. Journal of Sports Medicine and Physical Fitness, 45, 248-256.

Psotta, R., Bunc, V., Hendl, J., Tenney, D., \& Heller, J. (2011). Is repeated-sprint ability of soccer players predictable from field-based or laboratory physiological tests? Journal of Sports Medicine and Physical Fitness, 51, 18-25.

Quinney, H. A., Dewart, R., Game, A., Snydmiller, G., Warburton, D., \& Bell, G. (2008). A 26 year physiological description of a National Hockey League team. Applied Physiology, Nutrition and Metabolism, 33, 753-760.

Rampinini, E., Bishop, D., Marcora, S. M., Bravo, D. F., Sassi, R., \& Impellizzeri, F. M. (2007). Validity of simple field tests as indicators of match-related physical performance in top-level professional soccer players. International Journal of Sports Medicine, 28, 228-235.

Reed, A., Hansen, H., Cotton, C., Gauthier, R., Jette, M., Thoden, J., \& Wenger, H. (1979). Development and validation of an on-ice hockey fitness test. Canadian Journal of Applied Sport Science, 4, 245-252.

Reilly, T. (2005). An ergonomics model of the soccer training process. Journal of Sports Sciences, 23, 561-572.

Spencer, M., Bishop, D., Dawson, B., \& Goodman, C. (2005). Physiological and metabolic responses of repeated-sprint activities - specific to field-based team sports. Sports Medicine, 35, 1025-1044.

Spencer, M., Fitzsimons, M., Dawson, B., Bishop, D., \& Goodman, C. (2006). Reliability of a repeated-sprint test for field-hockey. Journal of Science and Medicine in Sport, 9, 181-184.

Vescovi, J. D., Murray, T. M., \& VanHeest, J. L. (2006). Positional performance profiling of elite ice-hockey players. International Journal of Sports Physiology and Performance, 1, 84-94.

Wragg, C. B., Maxwell, N. S., \& Doust, J. H. (2000). Evaluation of the reliability and validity of a soccer-specific field test of repeated sprint ability. European Journal of Applied Physiology, 83, 77-83. 